\title{
COUPLED CLOUDS AND CHEMISTRY OF THE GIANT PLANETS - A CASE FOR MULTIPROBES
}

\author{
SUSHIL K. ATREYA and AH-SAN WONG \\ Department of Atmospheric, Oceanic, and Space Sciences, The University of Michigan, Ann Arbor, \\ MI 48109-2143, USA
}

Received: 23 April 2004; Accepted in final form: 4 August 2004

\begin{abstract}
In seeking to understand the formation of the giant planets and the origin of their atmospheres, the heavy element abundance in well-mixed atmosphere is key. However, clouds come in the way. Thus, composition and condensation are intimately intertwined with the mystery of planetary formation and atmospheric origin. Clouds also provide important clues to dynamical processes in the atmosphere. In this chapter we discuss the thermochemical processes that determine the composition, structure, and characteristics of the Jovian clouds. We also discuss the significance of clouds in the big picture of the formation of giant planets and their atmospheres. We recommend multiprobes at all four giant planets in order to break new ground.
\end{abstract}

Keywords: Jupiter, giant planets; chemistry, clouds; chromophores, haze; multiprobes

That the Planets are not without Water, is made not improbable by the late Observations: For about Jupiter are observ'd some spots of a darker hue than the rest of his Body, which by their continual change show themselves to be Clouds: For the spots of Jupiter which belong to him, and never remove from him, are quite different from these, being sometimes for a long time not to be seen for these Clouds; and again, when these disappear, showing themselves. ...

Christianus Huygens, In Kosmotheoros 1698

\section{Introduction}

Ever since the invention of occhiale (telescope) by Galileo Galilei nearly four centuries ago, Jupiter's clouds have fascinated amateur astronomers and planetary scientists alike. With the availability of modern observing techniques, it has been possible to determine the morphology and characteristics of the upper visible clouds of Jupiter and Saturn. However, the cloud structure of the four giant planets is complex, extending deep into their atmospheres. Galileo Probe is the only entry probe ever deployed at a giant planet. But, the probe entered a meteorologically anomalous region - the Sahara Desert of Jupiter - one of the driest places on the planet. Hence, information on only the very tenuous clouds in the entry site could be gleaned from the Probe nephelometer measurements. These observations could not reveal anything about the cloud structure elsewhere on Jupiter. On the other hand, a good picture of the structure of Jovian clouds has begun to emerge by 
combining existing data (nephelometer, mass spectrometer, and remote imaging) with thermochemical models. The model predictions are for a three-layer cloud structure at Jupiter and Saturn, and a 4-5 layer cloud structure in the atmospheres of the icy giants, Uranus and Neptune. We will first discuss the cloud formation model, then compare the results with existing observations. We will then discuss the significance of clouds in the models of formation of the giant planets and their atmospheres, and finally make recommendations for future work.

\section{Thermochemical Cloud Model}

The equilibrium cloud condensation models (ECCM) of Jupiter date back to the pre-Voyager epoch. The model was first developed by Weidenschilling and Lewis (1973), and has undergone further development as described in Atreya and Romani (1985) and Atreya (1986). The lifting condensation level (LCL), i.e. the base of the cloud, is calculated by comparing the partial pressure $(e)$ and the saturation vapor pressure $\left(e_{\mathrm{c}}\right)$ of the condensible volatile. The LCL is reached at the altitude where relative humidity $\left(e / e_{\mathrm{c}}\right)$ of $100 \%$ is attained. The amount of condensate in the ECCM is determined by the temperature structure at the LCL and vicinity. The release of latent heat of condensation modifies the lapse rate, hence the temperature structure, of the atmosphere. Thus, the composition and structure of the clouds depend on the composition of the atmosphere, and in particular the distribution of condensible volatiles.

Thermochemical equilibrium considerations suggest that $\mathrm{NH}_{3}, \mathrm{H}_{2} \mathrm{~S}$ and $\mathrm{H}_{2} \mathrm{O}$ are the only species likely to condense in the atmospheres of Jupiter and Saturn, if the composition were solar. The presently known "elemental" abundance information for Jupiter, Saturn and the icy giants is given in Table 1, and also illustrated for Jupiter in Figure 1. As shown in Table I, $\mathrm{N}$ (from $\mathrm{NH}_{3}$ ) and $\mathrm{S}$ (from $\mathrm{H}_{2} \mathrm{~S}$ ) are enriched relative to solar, but $\mathrm{O}$ (from $\mathrm{H}_{2} \mathrm{O}$ ) is subsolar even at the deepest level in the region of the Galileo Probe entry on Jupiter. $\mathrm{O} / \mathrm{H}$ is expected to be enriched by a similar factor as the other heavy elements, i.e. $3 \pm 1$ (Owen et al., 1999; Atreya et al., 1999), since current ideas of the formation of Jupiter favor a core accretion model in which cold planetesimals are the original carriers of heavy elements (heavier than helium). If the heavy elements were delivered by clathrate hydrates, then the water abundance would be more than $9 \times$ solar in Jupiter's wellmixed atmosphere (Gautier et al., 2001a; b). In either case, condensation of water both as ice and droplets is inevitable.

For Saturn, tentative information on only one condensible species, $\mathrm{NH}_{3}$, is available, indicating perhaps a greater enrichment factor compared to that at Jupiter (Table I). In fact, $\mathrm{C} / \mathrm{H}$ also seems greater than at Jupiter. The progressively larger enrichment in the heavy elements from Jupiter to Neptune is consistent with the predictions of the core accretion model (although this fact is reflected also in Sat- 


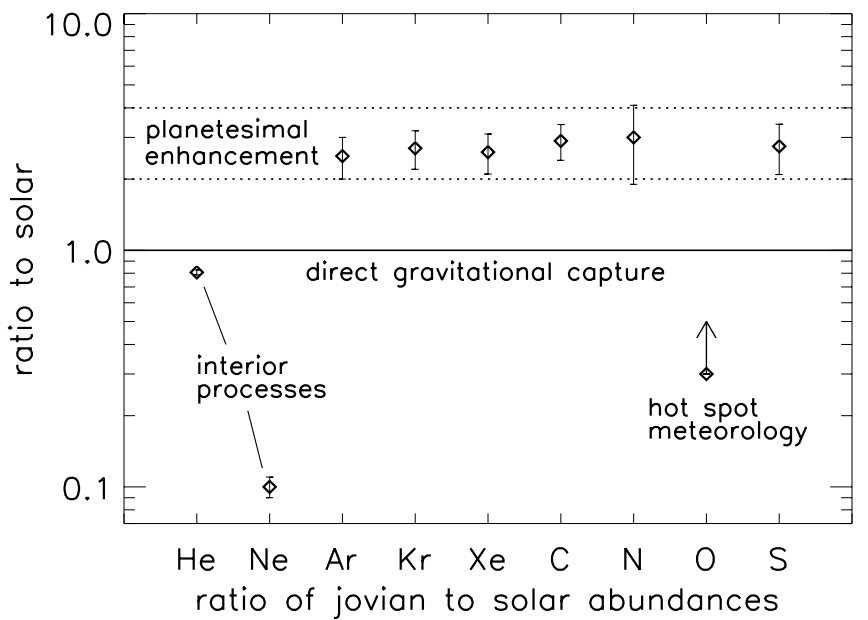

Figure 1. Elemental abundances (relative to $\mathrm{H}$ ) in Jupiter's atmosphere compared to the solar values. The Jupiter results are those measured by the Galileo Probe Mass Spectrometer (GPMS). Solid horizontal line shows that direct gravitational capture would result in elemental abundances (ratioed to $\mathrm{H}$ ), same as in the Sun. However, heavy elements, $\mathrm{Ar}, \mathrm{Kr}, \mathrm{Xe}, \mathrm{C}, \mathrm{N}$, and $\mathrm{S}$ are all found to be enriched by a factor of $3 \pm 1$. Figure updated from Owen et al. (1999).

TABLE I

Elemental and relevant isotopic abundances

\begin{tabular}{|c|c|c|c|c|c|}
\hline Elements & Sun & Jupiter/Sun & Saturn/Sun & Uranus/Sun & Neptune/Sun \\
\hline $\mathrm{He} / \mathrm{H}$ & 0.0975 & $0.807 \pm 0.02$ & $0.56-0.85$ & $0.92-1.0$ & $0.92-1.0$ \\
\hline $\mathrm{Ne} / \mathrm{H}$ & $1.23 \times 10^{-4}$ & $0.10 \pm 0.01$ & $?$ & 20-30 (?) & $30-50(?)$ \\
\hline $\mathrm{Ar} / \mathrm{H}$ & $3.62 \times 10^{-6}$ & $2.5 \pm 0.5$ & $?$ & 20-30 (?) & $30-50(?)$ \\
\hline $\mathrm{Kr} / \mathrm{H}$ & $1.61 \times 10^{-9}$ & $2.7 \pm 0.5$ & $?$ & 20-30 (?) & $30-50(?)$ \\
\hline $\mathrm{Xe} / \mathrm{H}$ & $1.68 \times 10^{-10}$ & $2.6 \pm 0.5$ & $?$ & $20-30(?)$ & $30-50(?)$ \\
\hline $\mathrm{C} / \mathrm{H}$ & $3.62 \times 10^{-4}$ & $2.9 \pm 0.5$ & $4-6$ & $20-30$ & $30-50$ \\
\hline $\mathrm{N} / \mathrm{H}$ & $1.12 \times 10^{-4}$ & $3.0 \pm 1.1 \mathrm{hs}$ & $2-4(?)$ & 20-30 (?) & $30-50(?)$ \\
\hline $\mathrm{O} / \mathrm{H}$ & $8.51 \times 10^{-4}$ & $0.29 \pm 0.1 \mathrm{hs}$ & $?$ & $20-30(?)$ & $30-50(?)$ \\
\hline $\mathrm{S} / \mathrm{H}$ & $1.62 \times 10^{-5}$ & $2.75 \pm 0.66 \mathrm{hs}$ & $?$ & 20-30 (?) & $30-50(?)$ \\
\hline $\mathrm{P} / \mathrm{H}$ & $3.73 \times 10^{-7}$ & 0.82 & $5-10$ & $20-30(?)$ & $30-50(?)$ \\
\hline Isotopes & Sun & Jupiter & Saturn & Uranus & Neptune \\
\hline${ }^{15} \mathrm{~N} /{ }^{14} \mathrm{~N}$ & $<2.8 \times 10^{-3}$ & $2.3 \pm 0.3 \times 10^{-3}$ & & & \\
\hline $\mathrm{D} / \mathrm{H} \times 10^{-5}$ & $2.1 \pm 0.5$ & $2.6 \pm 0.7$ & $2.25 \pm 0.35$ & $5.5_{-1.5}^{+3.5}$ & $6.5_{-1.5}^{+2.5}$ \\
\hline
\end{tabular}

After Atreya (2004). See also Atreya et al. (2003), Atreya et al. (1999) for references. O/H at Jupiter is from Wong et al. (2004a). The solar values are taken from Anders and Grevesse (1989) in order to maintain a standard reference. The heavy element ratios for Uranus and Neptune are taken to be the same as $\mathrm{C} / \mathrm{H}$ from $\mathrm{CH}_{4}$ measurements on these planets. Note: hs is 5-micron hotspot. 
urn's (5-10) $\times$ solar $\mathrm{P} / \mathrm{H}$, being a disequilibrium species, phosphine is not a good indicator of the heavy element enhancement factor). Thus, for purposes of cloud structure modeling, it is reasonable to assume a factor of 5 enhancement over solar for all of Saturn's condensible species, ammonia, ammonium hydrosulfide, and water.

The lapse rate, cloud structure and bases, and the cloud density in the outer planet atmospheres can be derived using thermodynamic equilibrium principles. The lapse rate depends both on latent heat released upon condensation and the heat of formation if a gas phase chemical reaction results into a condensate. Whenever the partial pressure of a constituent exceeds its saturated vapor pressure, condensation occurs. This process releases latent heat of condensation, thereby changing the local lapse rate. An expression for the wet adiabatic lapse rate $(\mathrm{d} T / \mathrm{d} z)$ can be derived using the principle of energy conservation for adiabatic expansion of a mole of gas.

Hydrogen sulfide does not condense by itself in the atmospheres of Jupiter and Saturn. In the gas phase, it can combine with ammonia to form ammonium hydrosulfide $\left(\mathrm{NH}_{4} \mathrm{SH}\right)$, or ammonium sulfide $\left(\left[\mathrm{NH}_{4}\right]_{2} \mathrm{~S}\right)$ which is less likely, i.e.,

$$
\mathrm{NH}_{3}(\mathrm{~g})+\mathrm{H}_{2} \mathrm{~S}(\mathrm{~g})=\mathrm{NH}_{4} \mathrm{SH} .
$$

With the inclusion of this "chemical condensation", the energy conservation equation (from first principle of thermodynamics) is

$$
d Q=0=\bar{C}_{P} d T-v d P+\sum_{k} L_{k} d X_{k}+L_{\mathrm{rx}} d X_{\mathrm{H}_{2} \mathrm{~S}}
$$

where $\bar{C}_{P}$ is mean molar heat capacity at constant pressure $P, \mathrm{~d} T$ is differential change in temperature, $v$ is molar volume of the gas, $\mathrm{d} X_{k}$ is the differential change in the number of moles of the $k^{\text {th }}$ gas due to condensation, $L_{k}$ is molar enthalpy of condensation (latent heat) of the $k^{\text {th }}$ gas, and $L_{\mathrm{rx}}$ is the molar heat of reaction/formation for the Reaction (1).

Ammonium hydrosulfide (or ammonium sulfide, if formed) would condense as a solid in the environmental conditions of all giant planets. The "chemical" condensation of $\mathrm{H}_{2} \mathrm{~S}$ results in the heat of formation, similar to the latent heat of condensation. The equilibrium constant, $K_{P}$, for Reaction (1) is given by:

$$
\log _{10} K_{P}=14.82-\frac{4705}{T}=\log _{10}\left(P_{\mathrm{NH}_{3}} P_{\mathrm{H}_{2} \mathrm{~S}}\right)
$$

from International Critical Tables, where $P_{k}$ is the partial pressure expressed in atmospheres and $T$ is the temperature in $K$. Also,

$$
P_{\mathrm{NH}_{3}}=P X_{\mathrm{NH}_{3}}, P_{\mathrm{H}_{2} \mathrm{~S}}=P X_{\mathrm{H}_{2} \mathrm{~S}}, d X_{\mathrm{NH}_{3}}=d X_{\mathrm{H}_{2} \mathrm{~S}}
$$


since the rate of concentration change of all species is the same in a mixed atmosphere. Differentiating Equation (3) and employing Equations (4) gives

$$
\frac{X_{\mathrm{H}_{2} \mathrm{~S}}+X_{\mathrm{NH}_{3}}}{X_{\mathrm{H}_{2} \mathrm{~S}} X_{\mathrm{NH}_{3}}} d X_{\mathrm{H}_{2} \mathrm{~S}}=10834 \frac{d T}{T^{2}}-2 \frac{d P}{P}
$$

Using the standard gas law $P v=R T$ (where $R$ is gas constant per mole), $\mathrm{d} P=-g \rho \mathrm{d} z$ (where $\rho=\bar{m} / v$ is mass density, and $\bar{m}$ is mean molecular weight), mixing ratio definition $X_{k}=P_{k} / P$, and Clausius-Clapeyron equation $\mathrm{d} P_{k} / \mathrm{d} T=$ $L_{k} / T \Delta v_{k} \sim L_{k} / T v_{k}$, we obtain

$$
\mathrm{d} X_{k}=\frac{1}{P} \mathrm{~d} P_{k}-\frac{P_{k}}{P^{2}} \mathrm{~d} P=X_{k}\left(\frac{L_{k} \mathrm{~d} T}{R T^{2}}+\frac{g \bar{m}}{R T} \mathrm{~d} z\right) .
$$

Substituting Equations (5) and (6) into Equation (2), the following expression for the wet adiabatic lapse rate of the atmosphere is obtained,

$$
\frac{\mathrm{d} T}{\mathrm{~d} z}=-\frac{\bar{m} g}{\overline{C_{P}}}\left(\frac{1+\frac{1}{R T}\left[\sum_{k} L_{k} X_{k}+\frac{2\left(X_{\mathrm{H}_{2} \mathrm{~S}} X_{\left.\mathrm{NH}_{3}\right) L_{\mathrm{rx}}}\right.}{X_{\mathrm{H}_{2}} \mathrm{~S}+X_{\mathrm{NH}_{3}}}\right]}{1+\frac{1}{\overline{C_{P} T^{2}}}\left[\sum_{k} \frac{L_{k}^{2} X_{k}}{R}+\frac{10834\left(X_{\mathrm{H}_{2} \mathrm{~S}} X_{\mathrm{NH}_{3}}\right) L_{\mathrm{rx}}}{X_{\mathrm{H}_{2}} \mathrm{~S}+X_{\mathrm{NH}_{3}}}\right]}\right)
$$

$\mathrm{NH}_{3}$ could also dissolve in $\mathrm{H}_{2} \mathrm{O}$, resulting in an "aqueous solution cloud" in the atmosphere. If the concentration of the solution is $C$ (due to $\mathrm{NH}_{3}$ condensation), then for every mole of solution, $(1-C)$ mole of $\mathrm{H}_{2} \mathrm{O}$ is condensed out, i.e.,

$$
\mathrm{d} X_{\text {solution }}=\frac{\mathrm{d} X_{\mathrm{H}_{2} \mathrm{O}}}{1-C} .
$$

In Equation (2), $L_{k} \mathrm{~d} X_{k}$ term should therefore reflect the heat due to condensation in solution, i.e.,

$$
\frac{L_{s} \mathrm{~d} X_{\mathrm{H}_{2} \mathrm{O}}}{(1-C)},
$$

where $L_{s}$ is the average heat of condensation of the solution. This will affect the lapse rate in Equation (7) and subsequently the atmospheric temperature.

The average density of the condensate $k$ between two closely spaced atmospheric levels $I$ and $J$ is given by

$$
\bar{D}=\frac{m_{k}\left(X_{k}^{I}-X_{k}^{J}\right) \bar{P}}{\bar{m} g \Delta z}
$$

where $m_{k}$ is the mass fraction of the $k^{\text {th }}$ condensate, $\Delta z$ is the height interval between the two levels, and $\bar{P}$ is the mean atmospheric pressure. 


\section{Cloud Model Results and Observations}

\subsection{Clouds OF JUPiter AND SATURN}

Based on the above considerations of equilibrium thermodynamics, it is possible to construct the ECCM for Jupiter and Saturn. Figure 2 shows results of ECCM calculations for Jupiter, with $1 \times$ solar and $3 \times$ solar condensible volatile abundances in the left panel, and greatly depleted condensible volatiles in the right panel in order to simulate the LCL of the clouds detected in the Galileo Probe Entry Site (PES). Since the Galileo Probe entered a dry region, the condensible volatiles were found to be greatly depleted to levels well below their expected condensation levels. It has been proposed that the depletion is caused by a giant downdraft extending to at least the 22 bar level or $160 \mathrm{~km}$ below 1 bar (Atreya et al., 1997; Owen et al., 1997; Atreya et al., 1999; Atreya et al., 2003), or a giant wave whose trough extends to at least the same level (Showman and Dowling, 2000). Neither of these hypotheses can completely explain the volatile distribution in the PES, thus additional investigation is needed. Note that the cloud densities calculated by the ECCM (Figure 2) represent upper limits and are much greater than any densities that would actually be expected in the Jovian atmosphere. This is due to the fact that atmospheric dynamics would not normally support a continuous wet adiabatic ascent through the entire atmospheric column, and microphysical processes lead to a reduction of the cloud density through precipitation. On the other hand, the ECCM is accurate in predicting the LCLs of the condensible volatiles, i.e. the cloud bases. This is clearly evident from a comparison of the ECCM calculations shown in Figure 2 and the observation of clouds.

The Galileo Probe Nephelometer detected a tenuous cloud layer at $1.3 \mathrm{bar}$, and more tenuous ones at 1.6 bar and 0.55 bar (Ragent et al., 1998). Although the $\mathrm{H}_{2} \mathrm{~S}$ and $\mathrm{H}_{2} \mathrm{O}$ mixing ratios in the PES at pressures less than 9 bar are unknown, their extrapolated values from the measured mixing ratios at pressures greater than 9 bar (Niemann et al., 1998; Atreya et al., 1999; Atreya et al., 2003), together with the $\mathrm{NH}_{3}$ profile inferred from the attenuation of Galileo Probe radio signal (Folkner et al., 1998), are consistent with those required to simulate the PES cloud bases (Figure 2). This is a strong evidence that the three cloud layers detected in the PES most likely represent the clouds of $\mathrm{NH}_{3}$-ice, $\mathrm{NH}_{4} \mathrm{SH}$-ice, and $\mathrm{H}_{2} \mathrm{O}$-ice, with their bases at, respectively, 0.5, 1.3, and 1.6 bar level (Atreya et al., 1999).

Outside the Galileo Probe entry site, only remote sensing observations exist. Extensive observations of Jupiter's upper visible clouds at relatively high spatial resolution were done with the Galileo orbiter imaging system at visible and near infrared wavelengths $(727,756,889 \mathrm{~nm})$. From an analysis of the low-mid latitude data, Banfield et al. (1998) identified a nearly ubiquitous cloud cover with its base at $750 \pm 200 \mathrm{mb}$, and cloud optical depth varying between 0 and 20. The atmospheric pressure range of the observed cloud bases is consistent with the range 


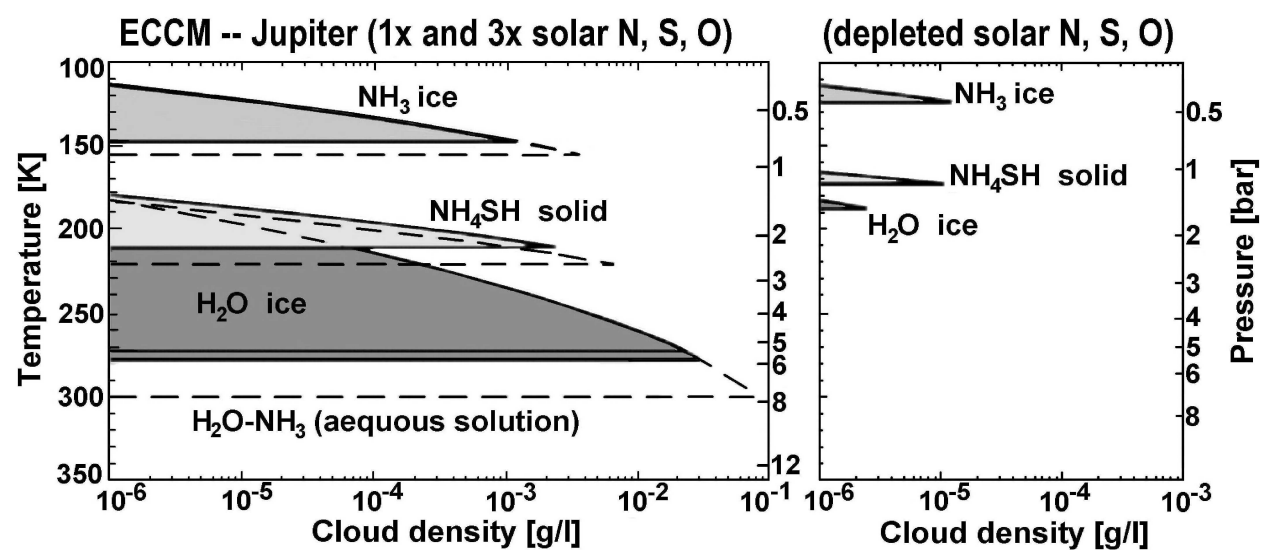

Figure 2. ECCM for Jupiter. Left panel: Elemental abundances of condensible volatiles are taken at $1 \times$ solar (solid area) and $3 \times$ solar (dashed lines) values. Right panel: as left panel, but with the following depleted condensible volatile abundances relative to solar: $\mathrm{H}_{2} \mathrm{O}: 0.01 \% ; \mathrm{NH}_{3}: 1 \% ; \mathrm{H}_{2} \mathrm{~S}$ : $0.5 \%$. The cloud concentrations (in grams per liter) represent upper limits. The temperature profile used in the ECCM is from Seiff et al. (1998) for the Galileo PES in right panel, and modified due to condensation in left panel. (After Atreya et al., 1999.)

predicted by the ECCM for $0.01 \times$ solar $\leq \mathrm{NH}_{3} \leq 4 \times$ solar, which spans the range of ammonia abundance on Jupiter, as measured by the Galileo Probe. The ECCM calculations show that ammonia would condense to ammonia ice at $\sim 500$ $\mathrm{mb}$ for $0.01 \times$ solar $\mathrm{N} / \mathrm{H}$, at $600 \mathrm{mb}$ for $0.5 \times$ solar $\mathrm{N} / \mathrm{H}$, at $720 \mathrm{mb}$ for $1 \times$ solar $\mathrm{N} / \mathrm{H}$, at $750 \mathrm{mb}$ for $1.2 \times$ solar $\mathrm{N} / \mathrm{H}$, at $840 \mathrm{mb}$ for $3 \times$ solar $\mathrm{N} / \mathrm{H}$, and at 1000 $\mathrm{mb}$ for $4 \times$ solar N/H (see Figure 2 for some cases). The Galileo Near Infrared Mapping Spectrometer (NIMS) near IR observations (Irwin et al., 2001) and the Galileo Probe and HST observations for the north equatorial belt (Sromovsky and Fry, 2002) indicate cloud opacity variations in the 1-2 bar region. This variability is most likely due to an ammonium hydrosulfide cloud predicted by the ECCM. The belts represent relatively dry, downwelling regions where the cloud locations are expected to be similar to those in the hot/warm spots.

The robustness of the ECCM is further strengthened by the observations of thunderstorms and lightning from the Galileo (Gierasch et al., 2000; Ingersoll et al., 2000) and Cassini (Dyudina et al., 2003) orbiters, attributed to the presence of water clouds deeper than 4-5 bars. Indeed, the ECCM calculations show that only water clouds can form in this pressure region (Fig. 2), and that at least $1 \times$ solar $\mathrm{H}_{2} \mathrm{O}$ is required for the cloud to be at $\geq 5$ bar level. But, the base of the water clouds cannot be determined by the above remote sensing observations. Hence the water abundance in well-mixed atmosphere of Jupiter is still unknown.

ECCM calculations for Saturn are shown in Figure 3, with the condensible volatiles taken as $1 \times$ solar and $5 \times$ solar. The $5 \times$ solar enhancement of the heavy elements is the more likely scenario for Saturn, as discussed in Section 2. Since the atmosphere of Saturn is colder relative to Jupiter, the condensation of the same 


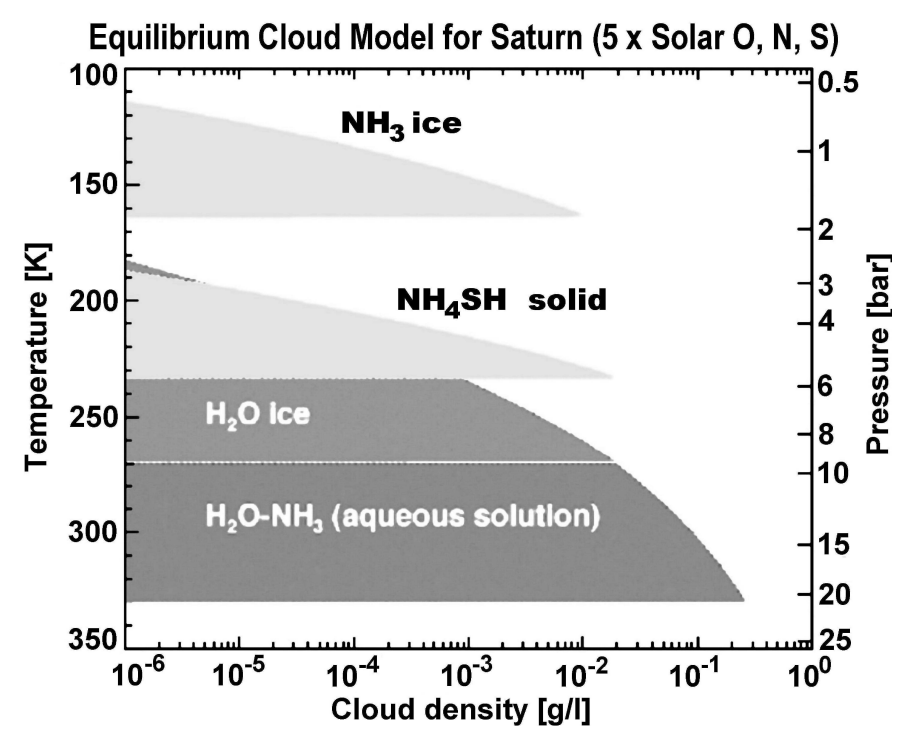

Figure 3. ECCM of Saturn, assuming a five-fold enrichment of the condensible volatiles, so that N/H, $\mathrm{S} / \mathrm{H}$, and $\mathrm{O} / \mathrm{H}$ are each $5 \times$ solar. The cloud concentrations (in gram/liter) represent upper limits.

species occurs at much greater pressure levels on Saturn compared to Jupiter. For example, with solar $\mathrm{O} / \mathrm{H}$, the base of the water cloud on Saturn (12.6 bars) is at nearly twice the pressure it is at Jupiter (5.7 bar); for $5 \times$ solar, the Saturn water cloud begins at 21 bars!

An important consideration of the cloud properties is the presence of a solution cloud on both planets. A droplet cloud due to an aqueous solution of ammonia in water becomes increasingly significant with greater and greater enrichment of ammonia and water, as can be seen for the $3 \times$ solar case for Jupiter and $5 \times$ solar case for Saturn (Figures 2 and 3). The solution cloud provides a ready loss mechanism for ammonia, followed by its loss in the ammonium hydrosulfide cloud higher up in the atmosphere. There are indications that even outside the hotspots the ammonia mixing ratio may be depleted by up to a factor of two down to the 2-3 bar level, i.e. below its expected condensation level (e.g., de Pater, 1986). The ammonia loss in $\mathrm{NH}_{4} \mathrm{SH}$ discussed here can explain some of this loss, but additional loss mechanisms may be necessary.

\subsection{Cloud Contaminants}

The agreement between the ECCM results on the locations of (i) all cloud layers in a dry region (Galileo PES), (ii) the upper cloud layers (Galileo imaging), and (iii) the purported water clouds (Galileo and Cassini imaging) is a strong evidence for ammonia ice as being the material of Jupiter's visible cloud layer. With the exception of relatively dry regions such as belts and hotspots, clouds 
are present everywhere on Jupiter. Yet, ammonia clouds have not been identified spectrally over most of the planet. In fact, Galileo/NIMS (Baines et al., 2002) and Cassini/CIRS (Composite Infrared Spectrometer; Wong et al., 2004a) observations find spectrally identifiable ammonia clouds only in certain locations, covering just $\sim 1 \%$ of Jupiter (Baines et al., 2002). In the case of Saturn, spectral identification of ammonia clouds is non-existent. It has been suggested previously that the lack of spectral identification of (ammonia) clouds on Jupiter and Saturn may be due to dusting by photochemical haze (Tomasko et al., 1984; West et al., 1986). In that case only the short-lived, freshly made plumes (Baines et al., 2002) or high altitude (ammonia) haze (Wong et al., 2004a) could remain uncontaminated and be identified spectrally as ammonia ice. Note that observations with the Infrared Space Observatory (ISO) in the 2.7-3 $\mu \mathrm{m}$ range also indicated the presence of spectrally-identifiable ammonia clouds on Jupiter (Brooke et al., 1998). However, the ISO data provide little information on the actual spatial distribution of the spectrally-identifiable ammonia clouds since the instrumental field-of-view covers some $60^{\circ}$ in latitude and $40^{\circ}$ in longitude (Baines et al., 2002). Another factor in the spectral obscuration of the upper visible cloud layer of these planets could be cloud properties, including ammonia aerosol particle size effects.

Throughout the atmospheres of Jupiter and Saturn, haze can be produced by photochemical processes. In the stratosphere, haze results primarily from condensation of polycyclic aromatic hydrocarbons (PAHs) and hydrazine $\left(\mathrm{N}_{2} \mathrm{H}_{4}-\mathrm{s}\right)$. The PAHs are produced in a series of hydrogen abstraction-acetylene addition (HACA) steps that involve, starting with $\mathrm{C}_{6} \mathrm{H}_{6}, \mathrm{H}$-abstraction (by $\mathrm{UV}$ photolysis, or reaction with $\mathrm{H}$ ) followed by $\mathrm{C}_{2} \mathrm{H}_{2}$-addition (Figure 4). Based on a coupled photochemical-aerosol microphysical model (Friedson et al., 2002; Wong et al., 2003) that includes the production rate of hydrocarbon haze particles, their sedimentation, growth and diffusion through the atmosphere, a hydrocarbon haze deposition rate of $\sim 10 \mu \mathrm{m}$ per year is calculated (one year is the average overturning time of the ammonia cloud particles; K. Baines, 2004, personal communication). The haze thickness is larger than the $3 \mu \mathrm{m}$ wavelength of observations (Baines et al., 2002). Thus, masking of the ammonium spectral signature by the grey hydrocarbon haze material is a distinct possibility.

In fact, the amount of the haze contaminant is expected to be much greater, since the stratospheric hydrocarbon haze would mix with the hydrazine haze particles produced in the photochemistry of ammonia in the region of the tropopause (Strobel, 1973; Atreya et al., 1977). Hydrazine haze is much more abundant than the hydrocarbon haze. A production rate of $6.9 \times 10^{10}$ hydrazine molecules $\mathrm{cm}^{-2} \mathrm{~s}^{-1}$ is calculated, which amounts to as much as $1.3 \mathrm{mg} / \mathrm{m}^{2}$ per Jovian day of the hydrazine condensate (Atreya et al., 1977). Being grayish, an admixture of the two can be an ideal contaminant for the ammonia clouds of Jupiter, rather than the white $\mathrm{N}_{2} \mathrm{H}_{4}$-s alone. The larger bulk of the admixture contaminant than of the PAH-condensate alone is also important for another reason-it can prevent spectral identification of 


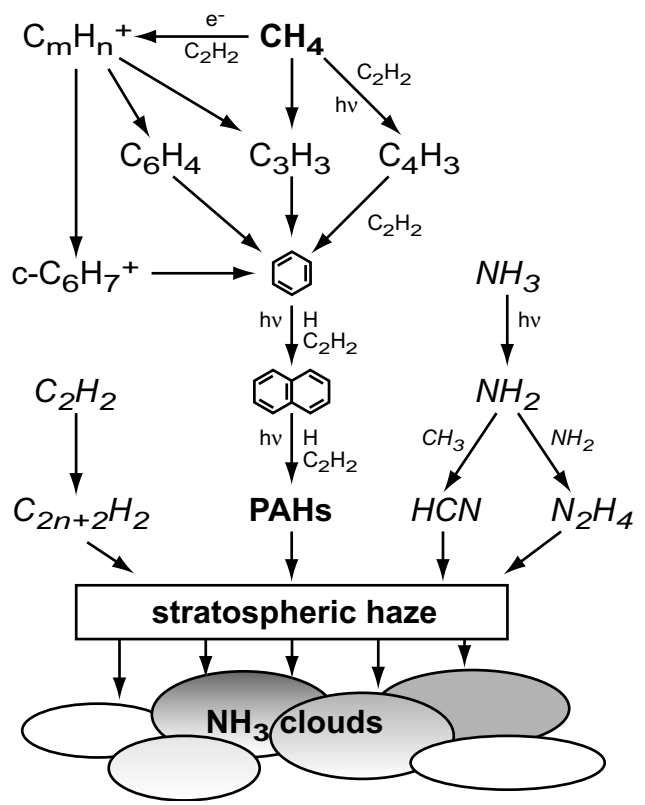

Figure 4. Illustration of important reaction pathways of haze formation on Jupiter. The pathways beginning with $\mathrm{C}_{2} \mathrm{H}_{2}$ and leading to polyyne polymerization (lower left branch) and the $\mathrm{HCN}$ polymerization (right branch) are minor contributors to haze formation. In the auroral regions of Jupiter, ion chemistry plays a decisive role in the hydrocarbon chemistry, so that nearly all of the "auroral" benzene (precursor to $\mathrm{PAH}$ ) is produced through the electron recombination of ring ion c- $\mathrm{C}_{6} \mathrm{H}_{7}^{+}$(Wong et al., 2003).

the clouds, even if the haze ends up in the core of $\mathrm{NH}_{3}$-ice particles, i.e. if it is suitable as condensation nuclei for ammonia. It has been suggested that spectral masking can occur if the contaminant is a significant fraction of the total mass (West et al., 1989), which is the case for the contaminant admixture. The same type of admixture is expected to be present in Saturn's atmosphere. Preliminary calculations show that the PAH produced hydrocarbon haze is abundant on Saturn also (Wong and Atreya, 2004), and the quantity of photochemically produced hydrazine is also fairly large (Atreya et al., 1980).

\subsection{Clouds of URAnus And Neptune}

We show in Figure 5 an ECCM model for Neptune. Uranus is nearly identical. The topmost cloud layer at $\sim 1$ bar level is made up of methane ice, according to the ECCM model. Voyager radio occultation observations did in fact infer a cloud layer at $\sim 1$ bar level. The base of the water-ice cloud for solar $\mathrm{O} / \mathrm{H}$ is expected to be at $\sim 40$ bar level, whereas for the $\mathrm{NH}_{3}-\mathrm{H}_{2} \mathrm{O}$ solution clouds it is at approximately twice this pressure. But, the heavy elements are most likely enriched relative to solar. The $\mathrm{C} / \mathrm{H}$ ratio at Uranus is $\sim 20-30 \times$ solar, and between $30 \times$ and $50 \times$ solar 

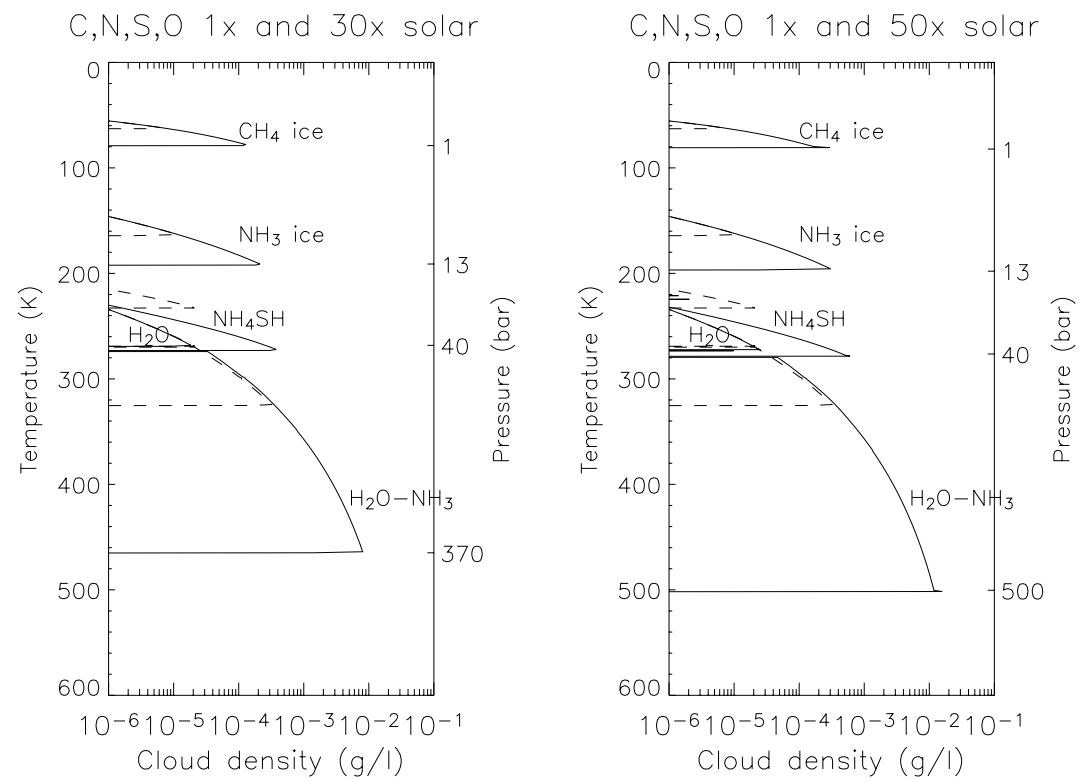

Figure 5. ECCM for Neptune, assuming $1 \times($ dashed lines) and $30 \times$ enrichment (left panel), and $1 \times\left(\right.$ dashed lines) and $50 \times$ enrichment (right panel), of condensible volatiles $\left(\mathrm{CH}_{4}, \mathrm{NH}_{3}, \mathrm{H}_{2} \mathrm{~S}\right.$, $\mathrm{H}_{2} \mathrm{O}$ ratioed to $\mathrm{H}$ ) relative to solar. Cloud bases for $30 \times$ solar, $50 \times$ solar cases are marked on right ordinates. The cloud concentrations (in gram/liter) represent upper limits. The structure and locations of the clouds at Uranus would be very similar due to similar thermal structure and atmospheric density in the tropospheres of the two planets.

at Neptune, as derived from the measurement of methane. As in the case of Jupiter, the enrichment factor for all other heavy elements is expected to be similar. Thus, $\mathrm{O} / \mathrm{H}$, hence water, as well as the other condensibles $\left(\mathrm{NH}_{3}, \mathrm{H}_{2} \mathrm{~S}\right)$ are also expected to be enhanced by factors of 20-30 or more relative to solar in the atmospheres of Uranus and Neptune. For purposes of illustration, we present cases with $1 \times, 30 \times$, and $50 \times$ solar enrichment of the condensible volatiles $\left(\mathrm{CH}_{4}, \mathrm{NH}_{3}, \mathrm{H}_{2} \mathrm{~S}, \mathrm{H}_{2} \mathrm{O}\right)$ in Figure 5 for Neptune. The $\mathrm{NH}_{3}-\mathrm{H}_{2} \mathrm{O}$ aqueous solution cloud base is calculated to be at 370 bars and 500 bars, respectively for $30 \times$ and $50 \times$ solar cases, accounting for van der Waals corrections. Some models (e.g. Ree, 1986; Podolak et al., 1991) predict the presence of a water-ammonia ionic ocean in the tens of kilobar region (depth depending upon $\mathrm{O} / \mathrm{H}$ and $\mathrm{N} / \mathrm{H}$ ). Such an ocean would be much deeper than the aqueous solution cloud of the ECCM discussed above. Therefore $\mathrm{NH}_{3}$ as well as $\mathrm{H}_{2} \mathrm{O}$ will have been depleted well below their ECCM condensation levels.

\subsection{Cloud Chromophores}

The brownish-orange-yellow colors of the visible clouds of Jupiter and Saturn continue to be a mystery, as there are no measurements available to determine the nature of the chromophore/s. Potential chromophores range from phosphorus 


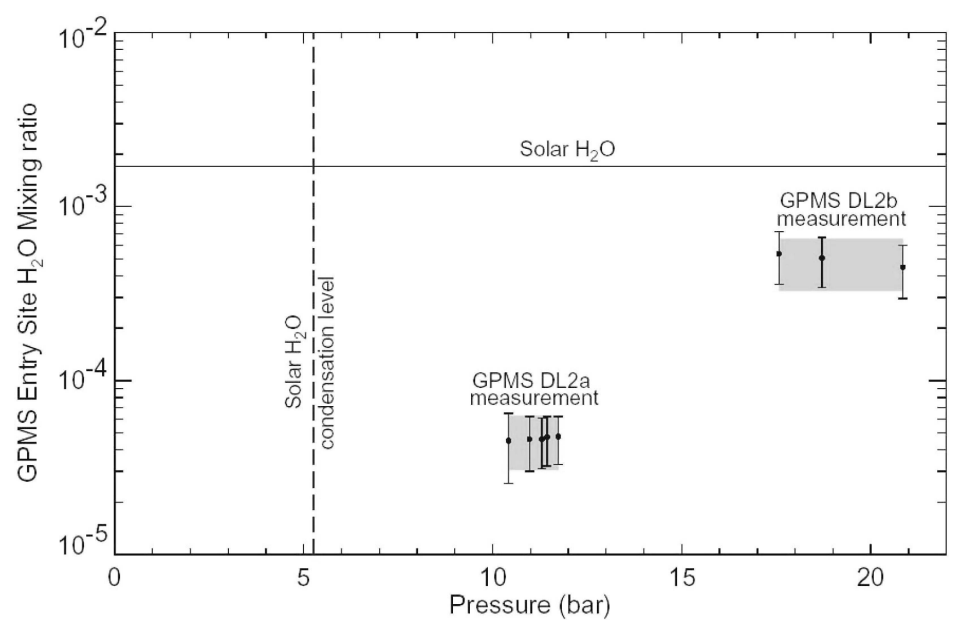

Figure 6. Galileo Probe Mass Spectrometer (GPMS) measured mixing ratio of water vapor as a function of atmospheric pressure. The mixing ratio increased dramatically, by nearly a factor of 10, between 10 and 22 bar levels, but remained subsolar even at the deepest level probed. The condensation level for solar water is shown by broken vertical line, i.e. the base of water ice cloud would be at approximately 5 bar. (After Atreya et al., 2003; Wong et al., 2004b.)

( $\mathrm{P}_{2}$-yellow; $\mathrm{P}_{4}$-red) produced in the stratospheric photochemistry of $\mathrm{PH}_{3}$, to sulfur species ( $\mathrm{S}_{8}$-yellow; ammonium polysulfide, $\left(\mathrm{NH}_{4}\right)_{x} \mathrm{~S}_{y}$-orange; hydrogen polysulfide, $\mathrm{H}_{x} \mathrm{~S}_{y}$-brown). In the atmospheres of Jupiter and Saturn, $\mathrm{H}_{2} \mathrm{~S}$ is sequestered in the $\mathrm{NH}_{4} \mathrm{SH}$ cloud in the 2 and 5 bar region, respectively, where the dissociating UV flux does not penetrate. Thus, the sulfur species are less likely to be responsible for the cloud colors, unless $\mathrm{H}_{2} \mathrm{~S}$ can be dissociated by other means, such as high energy cosmic rays. The aqua-marine color of Uranus and Neptune, on the other hand, is a direct consequence of the scattering of the Sun's bluish-green light by their atmospheres in which the Sun's red light is absorbed effectively by the large quantities of methane. The white clouds on these planets are due to the condensation of methane (Section 3.3).

\section{Future}

Much still remains mysterious about the clouds of the giant planets. It is only by having access to the region well below the main cloud layers that the abundances of key heavy elements can be determined. In the case of Jupiter, although the noble gases, $\mathrm{C}, \mathrm{N}$ and $\mathrm{S}$ have been measured in the well-mixed atmosphere, the water mixing ratio, hence the oxygen elemental abundance, has not been determined (Table I, Figure 1). The current status of the water measurement is shown in Figure 6. The cloud models discussed in Section 2 show that outside the hotspots, water clouds are expected at 5-8 bar level for $(1-3) \times$ solar $\mathrm{O} / \mathrm{H}$. But in the Galileo 
PES, the water mixing ratio was still rising at the deepest level probed, 22 bars! The thunderstorm and lightning observations on Jupiter (Section 2) are a good indication that the water abundance in the deep well-mixed atmosphere of Jupiter must be at least solar. But the results are not quantitative enough to be useful for constraining models of the formation of Jupiter and its atmosphere.

Since water is presumably the original carrier of heavy elements to Jupiter, it is the single most critical species remaining in order to constrain the formation models of Jupiter. It is not known at Jupiter, and, unfortunately, Cassini orbiter observations will not be able to yield the water abundance or, for that matter, the abundance of the heavy noble gases and sulfur in Saturn's atmosphere (there is a possibility of measuring $\mathrm{N} / \mathrm{H}$, however). Future missions that can accurately determine the water abundance in the deep well-mixed atmospheres of Jupiter and Saturn will be most valuable. Considering the possibility that water may be variable over these planets, it is imperative that measurements be carried out to depths well below the expected condensation levels of 5-20 bars, i.e. to pressures of at least 50100 bars. Since the meteorology of the hotspot entry sites is poorly understood, it is also important to carry out simultaneous measurements of other key species even if their mixing ratios were measured previously (e.g. at Jupiter by the Galileo Probe). Other supporting measurements for understanding the atmospheric composition data are winds and cloud particle properties.

A comparative study of the gas giants, when combined with a similar study for the icy giants, can provide the most comprehensive constraints on the models of formation of our solar system and, by extension, the extrasolar planetary systems. But determination of heavy element abundances on Uranus and Neptune becomes much more challenging than that at Jupiter and Saturn if the $\mathrm{O}$ and $\mathrm{N}$ elemental abundance must be measured also. This is because the water droplet cloud is expected to form at nearly a kilobar level - the kind of pressure encountered at the bottom of the Marianas Trench! But, in fact, even if the technological challenge of the probe and payload survival to and data transmission from kilobar levels, where the temperatures exceed $500 \mathrm{~K}$, could be overcome, the $\mathrm{O}$ and $\mathrm{N}$ elemental abundances may not be representative of their well-mixed atmospheric values due to the formation of an ionic solution in the tens of kilobar region, as discussed above. Fortunately, unlike Jupiter and Saturn, it is not imperative to measure the $\mathrm{N}$ and $\mathrm{O}$ abundance at Uranus and Neptune in order to constrain their formation models. The noble gases, $\mathrm{C}, \mathrm{S}, \mathrm{D} / \mathrm{H}$, and ${ }^{15} \mathrm{~N} /{ }^{14} \mathrm{~N}$ can provide all the constraints one needs for developing the models of the formation of the icy giants and their atmospheres. And these elements can be easily measured at shallower depths, i.e. at pressures of only 50-100 bars maximum. Unlike Jupiter and Saturn, neon is not expected to be depleted in the tropospheres of Uranus and Neptune, due to the absence of condensation of helium into droplets in which $\mathrm{Ne}$ dissolves, or by another fractionation process. In fact, the available measurements yield nearly solar $\mathrm{He} / \mathrm{H}$ in the atmospheres of Uranus and Neptune, unlike Jupiter (and possi- 
bly Saturn), as seen in Table 1 . Thus, $\mathrm{Ne} / \mathrm{H}$ is a critical measurement for Uranus and Neptune. Although the $\mathrm{N} / \mathrm{H}$ at Uranus and Neptune seems neither easy nor a good discriminator of models, the ${ }^{15} \mathrm{~N} /{ }^{14} \mathrm{~N}$ and $\mathrm{D} / \mathrm{H}$ isotope ratios are important measurements, as they would reveal the role of comets in the formation of these planets, by comparing the value against the cometary value and the "protosolar" ${ }^{15} \mathrm{~N} /{ }^{14} \mathrm{~N}$ and $\mathrm{D} / \mathrm{H}$ already measured at Jupiter by the Galileo Probe.

In summary, the big questions of the formation of the giant planets and the origin of their atmospheres require simultaneously addressing interrelated and intertwined issues of composition and clouds. It is essential to measure the elemental abundances below the cloud levels. For the gas giants, critical elements are: C, N, S, $\mathrm{O}, \mathrm{He}, \mathrm{Ne}, \mathrm{Ar}, \mathrm{Kr}, \mathrm{Xe}, \mathrm{D} / \mathrm{H}$ and ${ }^{15} \mathrm{~N} /{ }^{14} \mathrm{~N}$, whereas $\mathrm{N}$ and $\mathrm{O}$ are neither easily accessible nor critical for the icy giants. Supporting information on cloud structure and winds is essential also. Because of their disequilibrium nature, $\mathrm{PH}_{3}, \mathrm{AsH}_{3}, \mathrm{GeH}_{4}$ and $\mathrm{CO}$ are not good indicators of heavy element enhancement, nevertheless, their measurement on all giant planets is also desirable as they can reveal much about the interior processes. A comparative planetology approach can provide the best results. Multiple probes to the diverse multiple worlds of the giant planets are recommended for understanding the formation of our solar system and the extrasolar systems. Either in a single grand tour mission or on individual identical spacecraft missions, 2-3 probes deployed to 50-100 bars at each planet are recommended. A grand tour would fully exploit the capabilities of the Prometheus spacecraft, but celestial mechanics considerations might limit the mission to Jupiter, Saturn, and one of the two icy giants. In that case Neptune would be the ideal final target where, in addition to dropping the probes, a fully instrumented orbiter could also be deployed. A visionary approach involving multinational partnership is most desirable to accomplish the immensely challenging exploration of multiple planets by multiple probes. In the shorter term, relatively modest microwave spacecraft missions can help define the more ambitious probe mission/s to the giant planets.

\section{Acknowledgements}

We thank Toby Owen, Richard Young and Scott Bolton for illuminating discussions. SKA acknowledges support for this research by a grant from NASA's Planetary Atmospheres Program.

\footnotetext{
... And at the going off of these clouds, some spots have been taken notice of in him, much brighter than the rest of his Body, which remin'd but a little while, and then were hid from our sight. These Monsieur Cassini thinks are only the Reflection from the Snow that covers the tops of the Hills in Jupiter: but I should rather think that it is only the colour of the Earth, which chances to be free from those Clouds that commonly darken it.
} 


\section{References}

Anders, E. and Grevesse, N.: 1989, 'Abundances of the elements-Meteoritic and solar', Geochim. Cosmochim. Acta 53, 197-214.

Atreya, S.K., Donahue, T.M., and Kuhn, W.R.: 1977, 'The distribution of ammonia and its photochemical products on Jupiter', Icarus 31, 348-355.

Atreya, S.K., Kuhn, W.R., and Donahue, T.M.: 1980, 'Saturn: Tropospheric ammonia and nitrogen', Geophys. Res. Lett. 7, 474-476.

Atreya, S.K. and Romani, P.N.: 1985, 'Photochemistry and clouds of Jupiter, Saturn and Uranus', in G.E. Hunt (ed.), Planetary Meteorology, Cambridge University Press, pp. 17-68.

Atreya, S.K.: 1986, Atmospheres and Ionospheres of the Outer Planets and their Satellites, Chapter 3, Springer-Verlag, New York-Berlin.

Atreya, S.K., Wong, M.H., Owen, T.C., Niemann, H.B., and Mahaffy, P.R.: 1997, 'Chemistry and clouds of the atmosphere of Jupiter: A Galileo Perspective', in C. Barbieri et al. (eds.), Three Galileos: The Man, The Spacecraft, The Telescope, Kluwer, Dordrecht, The Netherlands, pp. 249-260.

Atreya, S.K., Wong, M.H., Owen, T.C., Mahaffy, P.R., Niemann, H.B., de Pater, I., Drossart, P., and Encrenaz, Th.: 1999, 'A comparison of the atmospheres of Jupiter and Saturn: deep atmospheric composition, cloud structure, vertical mixing, and origin', Planet. Space Sci. 47, 1243-1262.

Atreya, S.K., Mahaffy, P.R., Niemann, H.B., Wong, M.H., and Owen, T.C.: 2003, 'Composition and origin of the atmosphere-an update, and implications for the extrasolar giant planets', Planet. Space Sci. 51, 105-112.

Atreya, S.K.: 2004, 'Composition, clouds, and origin of Jupiter's atmosphere - a case for deep multiprobes into giant planets', ESA SP-544, 57-62.

Baines, K.H., Carlson, R.W. and Kamp, L.W.: 2002, 'Fresh ammonia ice clouds in Jupiter. I. Spectroscopic identification, spatial distribution, and dynamical implications', Icarus 159, 74-94.

Banfield, D., Gierasch, P.J., Bell, M., Ustinov, E., Ingersoll, A.P, Vasavada, A.R., West, R.A., and Belton, M.J.S.: 1998, 'Jupiter's cloud structure from Galileo imaging data', Icarus 135, 230-250.

Brooke, T.Y., Knacke, R.F., Encrenaz, Th., Drossart, P., and Crisp, D.: 1998, 'Models of the ISO 3- $\mu \mathrm{m}$ reflectance spectrum of Jupiter', Icarus 136, 1-13.

de Pater, I.: 1986, 'Jupiter's zone-belt structure at radio wavelengths', Icarus 68, 344-369.

Dyudina, U.A., Del Genio, A.D., Ingersoll, A.P., Porco, C., West, R.A., Vasavada, A.R., and Barbara, J.M.: 2003, 'Lightning on Jupiter observed in the $\mathrm{H}_{\alpha}$ line by the Cassini Imaging Science Subsystem', submitted to Icarus.

Folkner, W.M., Woo, R., and Nandi, S.: 1998, 'Ammonia abundance in Jupiter's atmosphere derived from attenuation of the Galileo probe's radio signal', J. Geophys. Res. 103, 22,847-22,856.

Friedson, A.J., Wong, A.S., and Yung, Y.L.: 2002, 'Models for polar haze formation in Jupiter's stratosphere', Icarus 158, 389-400.

Gautier, D., Hersant, F., Mousis, O., and Lunine, J.I.: 2001a, 'Enrichment in volatiles in Jupiter: A new interpretation of the Galileo measurements', Astrophys. J. 550, L227-L230.

Gautier, D., Hersant, F., Mousis, O., and Lunine, J.I.: 2001b, 'Erratum: Enrichment in volatiles in Jupiter: A new interpretation of the Galileo measurements', Astrophys. J. 559, L183.

Gierasch, P.J., Ingersoll, A.P., Banfield, D., Ewald, S.P., Helfenstein, P., Simon-Miller, A., Vasavada, A., Breneman, H.H., Senske, D.A., and the Galileo Imaging Team: 2000, 'Observation of moist convection in Jupiter's atmosphere', Nature 403, 628-630.

Huygens, C.: 1698, Kosmotheoros, English translation, 'The Celestial Worlds Discover'd: or, Conjectures Concerning the Inhabitants, Plants and Productions of the Worlds in the Planets', Frank Cass \& Co. Ltd., England, pp. 25-26. 
Ingersoll, A.P., Gierasch, P.J., Banfield, D., Vasavada, A.R., and the Galileo Imaging Team: 2000, 'Moist convection as an energy source for the large-scale motions in Jupiter's atmosphere', Science 403, 630-632.

Irwin, P.G.J., Weir, A.L., Taylor, F.W., Calcutt, S.B., and Carlson, R.W.: 2001, 'The origin of belt/zone contrasts in the atmosphere of Jupiter and their correlation with 5 - $\mu$ m opacity', Icarus 149, 397-415.

Niemann, H.B., et al.: 1998, 'The composition of the Jovian atmosphere as determined by the Galileo probe mass spectrometer', J. Geophys. Res. 103, 22,831-22,846.

Owen, T.C., Atreya, S.K., Mahaffy, P., Niemann, H.B. and Wong, M.H.: 1997, 'On the origin of Jupiter's atmosphere and the volatiles on the Medicean Stars', in C. Barbieri et al. (eds.), Three Galileos: The Man, The Spacecraft, The Telescope, Kluwer, Dordrecht, The Netherlands, pp. 289-297.

Owen, T., Mahaffy, P.R., Niemann, H.B., Atreya, S.K., Donahue, T., Bar-Nun, A., and de Pater, I.: 1999, 'A low-temperature origin for the planetesimals that formed Jupiter', Nature 402, 269-270.

Podolak, M., Hubbard W.B., and Stevenson D.J.: 1991, 'Models of Uranus interior and magnetic field', in J. Bergstralh, et al. (eds.), Uranus, The University of Arizona Press, Tucson, pp. 48-49.

Ragent, B., Rages, K.A., Knight, T.C.D., Arvin, P., and Orton, G.S.: 1998. 'The clouds of Jupiter: Results of the Galileo Jupiter mission probe nephelometer experiment', J. Geophys. Res. 103, 22,891-22,909.

Seiff, A., Kirk, D.B., Knight, T.C.D., Young, R.E., Mihalov, J.D., Young, L.A., Milos, F.S., Schubert, G., Blanchard, R.C. and Atkinson, D.: 1998, 'Thermal structure of Jupiter's atmosphere near the edge of a 5- $\mu \mathrm{m}$ hot spot in the north equatorial belt', J. Geophys. Res. 103, 22,857-22,889.

Ree, F.H.: 1986, 'A new approach to multiphase equilibria: Applications to high pressure physics problems', Physica 139-140B, 73-78.

Showman, A.P. and Dowling, T.: 2000, 'Nonlinear simulations of Jupiter's 5 micron hotspots', Science 289, 1737-1740.

Sromovsky, L.A. and Fry, P.M.: 2002, 'Jupiter's cloud structure as constrained by Galileo Probe and HST observations', Icarus 157, 373-400.

Strobel, D.F.: 1973, 'The photochemistry of $\mathrm{NH}_{3}$ in the Jovian atmosphere', J. Atmos. Sci. 30, 1205.

Tomasko, M.G., West, R.A., Orton, G.S., and Tejfel, V.G.: 1984, 'Clouds and aerosols in Saturn's atmosphere', in T. Gehrels and M.S. Matthews (eds.), Saturn, The University of Arizona Press, Tucson, pp. 150-194.

Weidenschilling, S.J. and Lewis, J.S.: 1973, 'Atmospheric and cloud structure of the Jovian planets', Icarus 20, 465-476.

West, R.A., Strobel, D.F., and Tomasko, M.G.: 1986, 'Clouds, aerosols, and photochemistry in the Jovian atmosphere', Icarus 65, 161-217.

West, R.A., Orton, G.S., Draine, B.T., and Hubbell, E.A.: 1989, 'Infrared absorption features for tetrahedral ammonia ice crystals', Icarus 80, 220-224.

Wong, A.S., Yung, Y.L., and Friedson, A.J.: 2003, 'Benzene and haze formation in the polar atmosphere of Jupiter', Geophys. Res. Lett. 30, 1447.

Wong, A.S. and Atreya, S.K.: 2004, 'Benzene, and other hydrocarbons on Saturn', EGS abstract, EGU04-A-02239, Nice, France.

Wong, M.H., Bjoraker, G.L., Smith, M.D., Flasar, F.M., and Nixon, C.A.: 2004a, 'Identification of the 10- $\mu \mathrm{m}$ ammonia ice feature on Jupiter', Planet. Space Sci. 52, 385-395.

Wong, M.H., Mahaffy, P.R., Atreya, S.K., Niemann, H.B., and Owen, T.C.: 2004b, 'Updated Galileo Probe Mass Spectrometer measurements of carbon, oxygen, nitrogen, and sulfur on Jupiter', Icarus, in press.

Address for Offprints: Sushil K. Atreya, Department of Atmospheric, Oceanic, and Space Sciences, The University of Michigan, Ann Arbor, MI 48109-2143, USA; atreya@umich.edu 